\title{
Kaon dispersion relation and flow in relativistic heavy-ion collisions
}

\author{
Bao-An Li and C. M. Ko \\ Cyclotron Institute and Physics Department, Texas A\&M University, College Station, Texas 77843
}

(Received 10 May 1996)

\begin{abstract}
Within the framework of a relativistic transport model (ART) for heavy-ion collisions at AGS energies, we examine the effects of a kaon dispersion relation on the transverse flow of kaons and their transverse momentum and azimuthal angle distributions. We find that the transverse flow is the most sensitive observable for studying the kaon dispersion relation in dense medium. [S0556-2813(96)02412-0]
\end{abstract}

PACS number(s): 25.75.Ld, 13.75.Jz, 21.65.+f

The properties of a kaon in the extremely hot and dense enviroment created in relativistic heavy-ion collisions have been the subject of many theoretical studies (see, e.g., [1] for a recent review). Experimentally, to extract the in-medium kaon properties is one of the most challenging tasks at several heavy-ion facilities (e.g., [2,3]). In a recent study it was first proposed that the kaon collective flow might be a sensitive probe of the kaon dispersion relation in a dense medium [4]. Furthermore, within the relativistic Valsov Uehling-Uhlenbeck (RVUU) model [5] it was shown that for heavy-ion collisions at SIS/GSI energies $(E / A \leqslant 2 \mathrm{GeV})$ the flow pattern of kaons is affected dramatically by the kaon potential in medium [4,6]. This suggestion has recently attracted much attention [7]. In particular, at SIS/GSI significant efforts will be devoted in the next few years to experimental studies of kaon flow and the extraction of the kaon dispersion relation in medium [8]. It is an interesting question; we were actually frequently asked at recent meetings whether the effect also exists at higher energies. In this work, we extend the study to AGS energies and show that at high energies the kaon flow is also a very sensitive probe of the kaon dispersion relation. For comparison we also examine effects of the kaon dispersion relation on its transverse momentum distribution and azimuthal angle distribution with respect to the reaction plane ("squeezeout"). Results of this study are expected to be useful for current experiments at Brookhaven's AGS (e.g., E859, E866 and E877 Collaborations) and the long range plan of GSI.

Our study is based on a relativistic transport model (ART) for heavy-ion collisions at AGS energies [9]. In this model the phase space distribution functions of baryons $\left[N, \Delta(1232), N^{*}(1440), N^{*}(1535), \Lambda, \Sigma\right]$ and mesons $(\pi, \rho, \omega, \eta, K)$ are evolved under the influence of hadronhadron scatterings and also optional mean fields. In transport models (see, e.g., [10-12] for a review), the imaginary part of a kaon self-energy is approximately treated by the scatterings of kaons with other hadrons, and the real part of the self-energy is given by the mean-field potential. Although various approaches have been used to evaluate the kaon dispersion relation in a dense medium (e.g., [1,13-19]), we shall use in the present study as an illustration the kaon dispersion relation determined from the kaon-nucleon scattering length $a_{K N}$ using the impulse approximation [1], i.e.,

$$
\omega\left(p, \rho_{b}\right)=\left[m_{K}^{2}+p^{2}-4 \pi\left(1+\frac{m_{K}}{m_{N}}\right) a_{K N} \rho_{b}\right]^{1 / 2},
$$

where $m_{K}$ and $m_{N}$ are the kaon and nucleon masses, respectively; $\rho_{b}$ is the baryon density and $a_{K N} \approx-0.255 \mathrm{fm}$ is the isospin-averaged kaon-nucleon scattering length. The kaon potential in medium can then be defined as

$$
U\left(p, \rho_{b}\right)=\omega\left(p, \rho_{b}\right)-\left(m_{K}^{2}+p^{2}\right)^{1 / 2} .
$$

Effects of the above potential on experimental observables can be seen qualitatively from the force acting on a kaon during its propogation

$$
\vec{F}=\frac{d \vec{p}}{d t}=-\vec{\nabla} U=\frac{2 \pi}{\omega}\left(1+\frac{m_{K}}{m_{N}}\right) a_{K N} \vec{\nabla} \rho_{b}
$$

Since the force is inversely proportional to the energy $\omega$, low energy particles are most likely to be affected by the kaon potential. This suggests that one should look for signatures of the kaon potential on kaon observables mainly around the midrapidity. Moreover, the kaon potential tends to force kaons away from the direction along which the baryon density

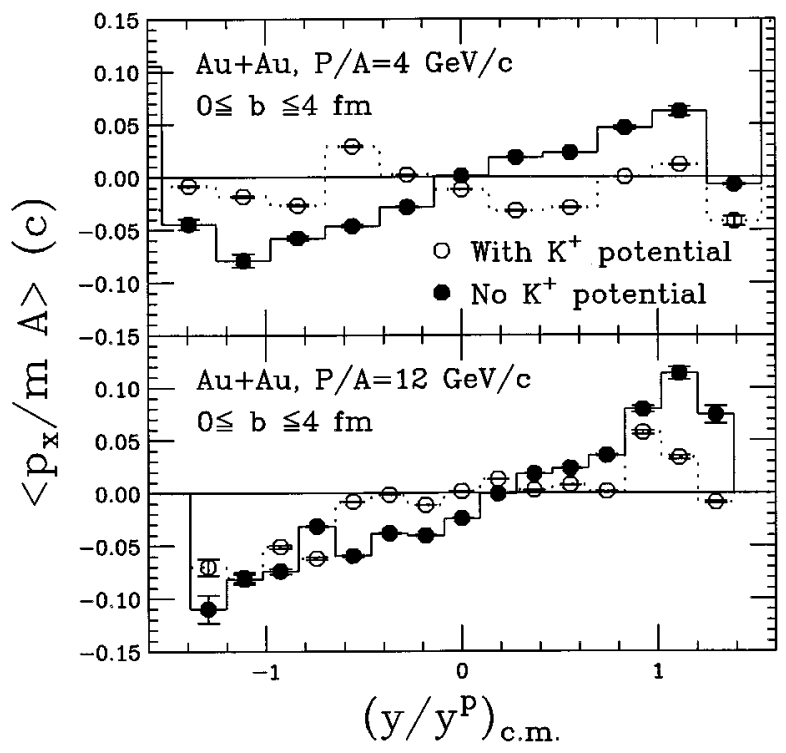

FIG. 1. The average transverse momentum of kaons in the reaction plane for $\mathrm{Au}+\mathrm{Au}$ reactions at $P_{\text {beam }} / A=4 \mathrm{GeV} / c$ (upper window) and $12 \mathrm{GeV} / c$ (lower window) at impact parameters less than $4 \mathrm{fm}$. The open (filled) circles are the results obtained with (without) the kaon potential. 

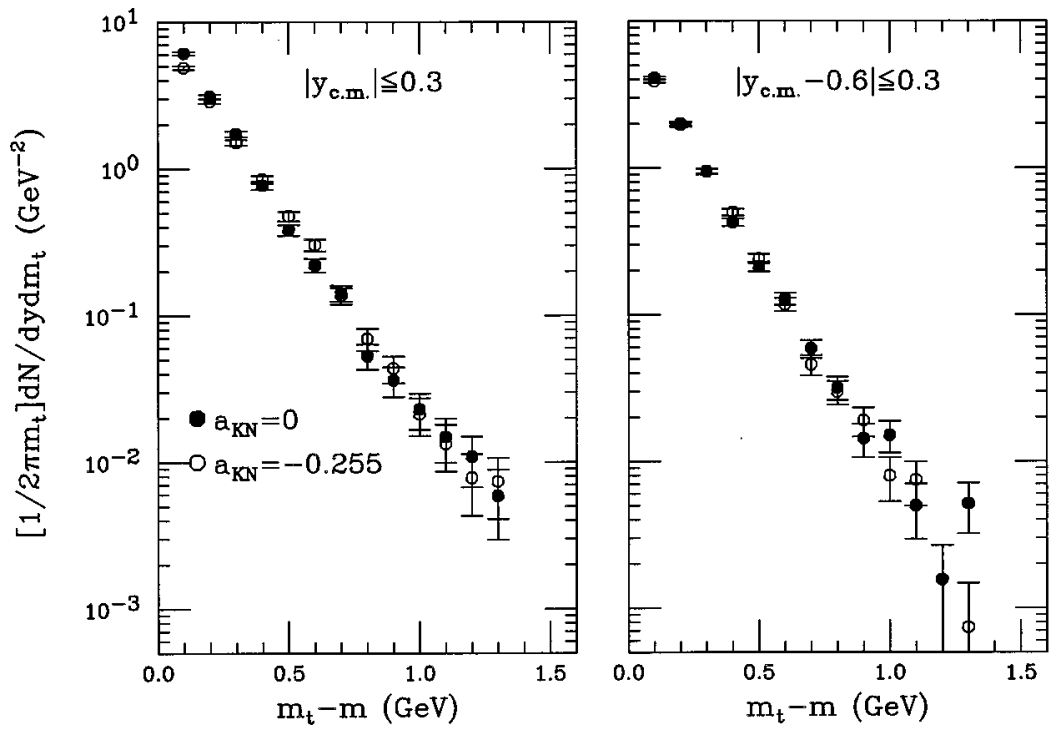

FIG. 2. Inclusive kaon transverse momentum distributions around midrapidity (left window) and projectile rapidity (right window) for the reaction of $\mathrm{Au}+\mathrm{Au}$ at $P_{\text {beam }} / A=4 \mathrm{GeV} / c$ and impact parameters less than $4 \mathrm{fm}$. The open (filled) circles are the results obtained with (without) the kaon potential.

increases. The latter reveals the underlying reason why the kaon transverse flow is a sensitive probe of its potential in medium. The transverse flow of baryons in heavy-ion collisions is an experimentally well-established fact at energies even as high as $160 \mathrm{GeV} /$ nucleon. Kaons gain some collectivity not only from their interactions with baryons, but also because they are mainly produced from baryon-baryon collisions in heavy-ion collisions. The baryon transverse flow results in a large asymmetry of baryon density distribution in the reaction plane, especially in the later stage of the reaction. Kaons moving in this medium thus develop an opposite asymmetry due to their interactions with baryons through the mean-field potential. To demonstrate this effect we will compare in the following results obtained with and without the kaon potential.

It is now well known that the best way to reveal transverse flow in heavy-ion collisions is to perform the in-plane transverse momentum analysis [20]. We have recently performed this analysis for baryons and pions using the ART model for heavy-ion collisions at beam energies from SIS/ GSI to AGS/Brookhaven energies [9]. Here we concentrate on the analysis for kaons. In Fig. 1 the average kaon transverse momentum (scaled by kaon mass) in the reaction plane is shown as a function of rapidity (scaled by the beam rapidity) for $\mathrm{Au}+\mathrm{Au}$ reactions at impact parameters smaller than $4 \mathrm{fm}$ (typical central collisions) and beam momentum of 4 $\mathrm{GeV} / c$ (upper window) and $12 \mathrm{GeV} / c$ (lower window). Experiments at these energies have already been done, but no kaon flow analysis has been carried out yet. The filled circles are the results without any mean-field potential for kaons. Comparing these to our early studies of nucleons and pions we see that kaons flow in the same direction as nucleons and pions. The magnitude of the average kaon transverse momentum in the reaction plane seems to be between that of nucleons and pions. More quantitatively, in the case of $P / A=12 \mathrm{GeV} / c$, the average transverse momentum at the projectile rapidity is about $0.12,0.04$, and $0.01 \mathrm{GeV} / c$ for nucleons, kaons, and pions, respectively. With the kaon potential the flow pattern of kaons changes dramatically, especially around the midrapidity as one expects. This observation is in agreement with that found at lower energies $[4,6]$, i.e., the kaon potential reduces the kaon flow or even changes its direction. This strong dependence on the kaon potential makes the kaon flow analysis a valuable tool to study the kaon dispersion relation in medium.

To see more clearly the advantage of the kaon flow analysis over traditionally measured single-particle observables in studying the kaon dispersion relation, we examine in Fig. 2 the kaon transverse momentum distribution around the midrapidity (left window) and projectile rapidity (right window) for the reaction of $\mathrm{Au}+\mathrm{Au}$ at $P / A=4 \mathrm{GeV} / c$ and im-

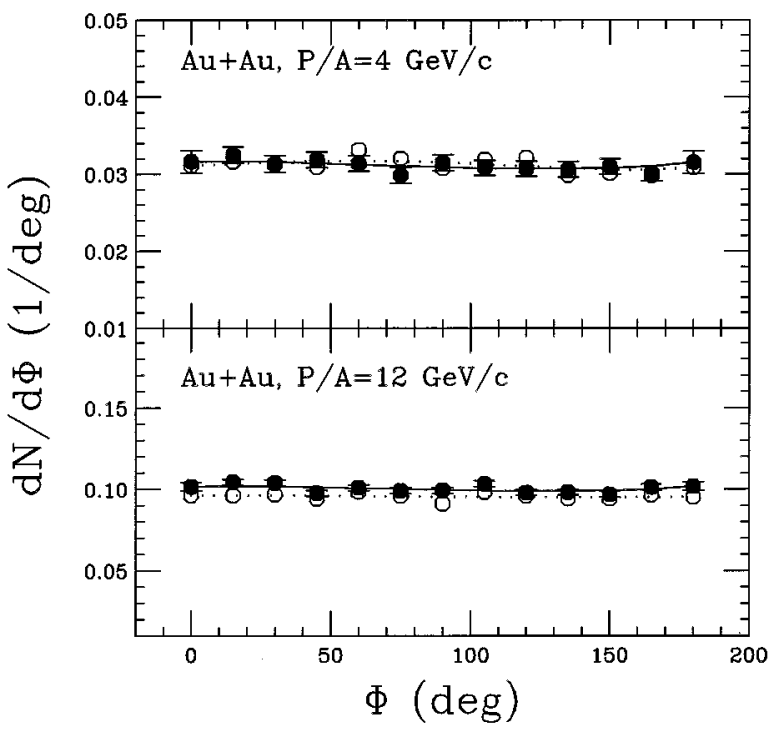

FIG. 3. The azimuthal angle distribution of kaons with respect to the reaction plane in the $\mathrm{Au}+\mathrm{Au}$ reactions described in Fig. 1. 
pact parameters less than $4 \mathrm{fm}$. There seems to be no obviously measurable effect of the kaon potential on the inclusive spectra in both rapidity ranges. Similar results have also been seen at $P / A=12 \mathrm{GeV} / c$ and the calculations agree well with the E866/E802 data as we have shown in [9]. The observation that the kaon flow, but not the inclusive spectrum, is sensitive to the kaon potential, is very similar to that observed for nucleons in heavy-ion collisions at Bevalac, SIS, and AGS energies [9]. The main reason for the insensitivity of the inclusive spectrum to the mean-field potential is that the transverse collective flow velocity or the change of kaon momentum due to the mean-field potential, is too small compared to the average thermal velocity of particles to be seen in the inclusive spectrum. For this reason it has been recently planned to measure the spectrum ratio of $K^{+}$over $K^{-}$[22], where enhanced effects of $K^{+}$and $K^{-}$potentials, which are opposite in sign due to the $G$ parity, might show up more clearly [21].

In heavy-ion collisions at lower energies the azimuthal angle distribution $(d N / d \phi)$ of particles with respect to the reaction plane has been used to identify a possible collectivity perpendicular to the reaction plane. The enhanced emission of particles in this direction has been dubbed the "squeezeout" phenomenon. For $\mathrm{Au}+\mathrm{Au}$ reactions it was shown in Ref. [23] that for both nucleons and pions the strength of the squeezeout decreases as the beam energy increases and almost disappears at energies higher than about 2 $\mathrm{GeV} /$ nucleon due to the increased global thermalization of the reaction system. At AGS energies one thus expects kaons to have a very small collectivity perpendicular to the reaction plane. Indeed, we find, as shown in Fig. 3 for the reaction of $\mathrm{Au}+\mathrm{Au}$ at $P / A=4$ and $12 \mathrm{GeV} /$ nucleon, that there is no observable kaon squeezeout from the calculations. Consequently, the azimuthal angle distribution with respect to the reaction plane is insensitive to the kaon potential. At lower energies, however, the situation might be different [24].

In summary, within the framework of a relativistic transport model (ART) for heavy-ion collisions at AGS energies, we have examined the effects of the kaon dispersion relation on the kaon transverse flow, transverse momentum distribution, and azimuthal angle distribution. It is found that the transverse flow is the most sensitive observable for studying the kaon dispersion relation in a dense medium.

We would like to thank D. Best and G. Q. Li for useful discussions. This work was supported in part by NSF Grant No. PHY-9509266.
[1] G.E. Brown, C.-H. Lee, M. Rho, and V. Thorsson, Nucl. Phys. A567, 937 (1994).

[2] E. Grosse, Prog. Part. Nucl. Phys. 30, 89 (1993).

[3] J. Stachel et al., Nucl. Phys. A566, 183c (1994).

[4] G.Q. Li, C.M. Ko, and B.A. Li, Phys. Rev. Lett. 74, 235 (1995).

[5] C.M. Ko, Q. Li, and R. Wang, Phys. Rev. Lett. 59, 1084 (1987); C.M. Ko and Q. Li, Phys. Rev. C 37, 2270 (1988).

[6] G.Q. Li and C.M. Ko, Nucl. Phys. A594, 460 (1995).

[7] FOPI Collaboration, J.L. Ritman et al., Z. Phys. A 352, 355 (1995).

[8] V. Metag, et al., private communication.

[9] B.A. Li and C. M. Ko, Phys. Rev. C 52, 2037 (1995); 53, R22 (1996); Nucl. Phys. A601, 457 (1996).

[10] Q. Li, J.Q. Wu, and C.M. Ko, Phys. Rev. C 39, 849 (1989).

[11] S.J. Wang, B.A. Li, W. Bauer, and J. Randrup, Ann. Phys. (N.Y.) 209, 251 (1991).

[12] W. Bauer, Prog. Part. Nucl. Phys. 30, 45 (1993).

[13] D.B. Kaplan and A.E. Nelson, Phys. Lett. B 175, 57 (1986);
A.E. Nelson and D.B. Kaplan, ibid. 192, 193 (1987).

[14] G.E. Brown, K. Kubodera, and M. Rho, Phys. Lett. B 193, 273 (1987).

[15] H.D. Politzer and M.B. Wise, Phys. Lett. B 273, 156 (1991).

[16] T. Muto and T. Tatsumi, Phys. Lett. B 283, 165 (1992).

[17] G. E. Brown, C.M. Ko, and K. Kubodera, Z. Phys. A 341, 301 (1992).

[18] M Lutz, A. Steiner, and W. Weise, Phys. Lett. B 278, 29 (1992).

[19] H. Yabu, S. Nakamura, F. Myhrer, and K. Kubodera, Phys. Lett. B 315, 17 (1983).

[20] P. Danielewicz and G. Odyniec, Phys. Lett. 157B, 146 (1985).

[21] X.S. Fang, C.M. Ko, G.E. Brown, and V. Koch, Phys. Rev. C 47, 1678 (1993).

[22] K.L. Wolf, private communication.

[23] B.A. Li, Phys. Lett. B 319, 412 (1993); Nucl. Phys. A570, 797 (1994).

[24] G.Q. Li, C.M. Ko, and G.E. Brown, Phys. Lett. B, in press. 